Relations industrielles

Industrial Relations

\title{
Les sciences sociales et l'homme
}

\section{Antonio Barrette}

Volume 4, numéro 2, octobre 1948

URI : https://id.erudit.org/iderudit/1023434ar

DOI : https://doi.org/10.7202/1023434ar

Aller au sommaire du numéro

Éditeur(s)

Département des relations industrielles de l’Université Laval

ISSN

0034-379X (imprimé)

1703-8138 (numérique)

Découvrir la revue

Citer cet article

Barrette, A. (1948). Les sciences sociales et l'homme. Relations industrielles / Industrial Relations, 4(2), 17-18. https://doi.org/10.7202/1023434ar
Résumé de l'article

Nous sommes heureux de publier le texte de l'allocution présentée par l'honorable Antonio Barrette, ministre du Travail de la province de Québec, lors de la remise d'un doctorat d'honneur en sciences sociales, à l'Université de Montréal, le 28 mai 1948 .
Tous droits réservés @ C Département des relations industrielles de l’Université Laval, 1948
Ce document est protégé par la loi sur le droit d'auteur. L’utilisation des services d'Érudit (y compris la reproduction) est assujettie à sa politique d'utilisation que vous pouvez consulter en ligne.

https://apropos.erudit.org/fr/usagers/politique-dutilisation/ 


\title{
LES SCIENCES SOCIALES ET L'HOMME
}

\author{
Honorable Antonio Barkette
}

\author{
Nous sommes heureux de publier le texte de l'allocution présentée par l'honorable Antonio Barrette, \\ ministre du Travail de la province de Québec. lors de la remise d'un doctorat d'honneur \\ en sciences sociales, à l'Université de Montréal, le 28 mai 1948.
}

« Beaucoup de savants de l'époque moderne \$, écrit le Prince Louis de Broglie, evictimes d'un réalisme naïf, ont adopté, presque sans s'en apercevoir, une certaine nétaphysique à caractère matérialiste et mécaniste, et l'ont considérée comme l'expression même de la pensée scientifique s. $^{1}$

Comme il est réconfortant d'entendre l'un des plus ̨rands physiciens vivants venir libérer les sciences positives du collier de fer, où si longtemps, le matérialisme les a enfermées. Nous sommes particulièrement bien placés, dans cette Maison, pour comprendre la valeur et la portée d'une telle libération, nous qui ne pouvons pas concevoir que les sciences sociales reposent sur d'autres postulats que ceux que nous savons être la Vérité Révélée elle-même. Car nous édifions notre recherche sur ce que nous professons dans notre vie, c'est-à-dire que c'est une personne humaine, au sens où l'Eglise l'enseigne, qui est présente en chacun des membres du corps social.

C'est en fonction de cette personne humaine que nous nous efforcerons de comprendre les phénomènes sociaux; c'est en fonction d'elle que nous utiliserons les résultats de notre science pour l'action; c'est pour elle que nous construirons la Cité. Et, de même qu'on juge l'arbre à ses fruits, de même que l'on apprécie les calculs d'un ingénieur à la solidité du pont qu'il construit, de même nous proposerons à nos frères du monde entier de juger de la valeur de notre postulat sur l'harmonie et le bonheur de notre société. Nous leur dirons que le bonheur de nos familles, nous leur dirons que notre paix sociale, très imparfaite encore, sans doute, mais combien plus réelle qu'ailleurs, sont les fruits d'une science sociale dont le postulat fondamental est en accord profond avec la Révélation, mieux encore, qu'il est tiré d'Elle. Peut-être alors, et Dieu aidant, les yeux de certains d'entre eux s'ouvriront-ils, parce que nous aurons tenté, de bonne foi, et dans l'humilité, de jouer pleinement notre rôle de charité au sein du monde, notre rôle qui est de porter témoignage.

Ayant ainsi affirmé notre volonté de voir la personne humaine au centre même des sciences sociales, nous nous efforcerons de définir de façon précise le contenu de ces deux mots.

\section{* * * *}

L'Homme se révèle au sociologue comme un fait concret qu'il faut prendre et qu'il faut comprendre. Parmi tous les phénomènes humains qui sollicitent l'étude, se présentant sous mille aspects, comment choisir, et sur- tout, comment classer? Selon le mot profond d'Henri Poincaré: «on fait la science avec des faits comme une maison avec des pierres, mais une accumulation de faits n'est pas plus une science qu'un tas de pierres n'est une maison $\gg .^{2}$ C'est donc pour déterminer comment

les faits humains permettent de reconstituer l'homme que lo postulat est nécessaire, et le résultat sera différent, selon que l'on définira la conscience comme une émanation de la matière, corruptible et périssable au même degré qu'elle, ou comme une réalité spirituelle, libre et immortelle. Choisissant donc de prendre les vérités révélées de notre Foi comme postulats d'observation de notre science, nous serons conduits à distinguer, en sociologie comme en économique et en politique, les trois réalités dont la hiérarchie même forme l'unité de la personne humaine: la réalité biologique, la réalité émotionnelle, la réalité spirituelle.

Réalité biologique, d'àbordl Car nous croyons que l'homme est un esprit incarné et nous croyons aussi à la résurrection de la chair. Et nous observerons rapidement que c'est à propos des exigences banales de la réalité biologique que se poseront les problèmes parfois les plus complexes de la vie économique, et, par conséquent, de la science économique. Exigence de l'alimentation qui doit, selon les progrès actuels de la diététique, être non seulement hygiénique et suffisante, mais aussi appétissante. Exigence du vêtement qui remplit une triple mission morale, hygiénique et esthétique. Exigence de l'habitation qui, au regard de la réalité biologique, doit répondre au triple souci de la protection thermique, de l'aération et de la lumière. Exigence du repos dont l'étude, de plus en plus poussée à l'occasion des relations industrielles, a montré l'importance considérable tant du point de vue de la santé que de celui du rendement. Exigence, aussi, de la sécurité dont le monde du travail éprouve le besoin avec tant d'acuité, qu'il s'agisse de la sécurité en cas de maladie ou de la sécurité de la vieillesse. Exigence enfin qui résulte de la fécondité même de l'amour humain et d'où dérive la volonté de fonder une famille. Toutes ces exigences de la réalitẻ biologique méritent une étude précise, déjà très largement commencée, par chaque discipline particulière et dont il appartient aux sciences sociales d'utiliser la synthèse.

Réalité émotionnelle, ensuitel Car aux frontières de la chair et de l'esprit, c'est toute la sensibilité qui est à l'origine de tant de problèmes, familiaux et sociaux! C'est la sensibilité qui tyrannise et passionne de façon si profondément physique et qui pourtant demeure si clairement spirituelle dans ses élans et dans ses espoirsl C'est le

(2) H. Ponncant, La science et Phypothese, p. 178. 
besoin d'affection, le besoin d'être aimé et apprécié, non seulement en famille, mais aussi au travail, et dont les plus récentes observations des relations industrielles ont établi l'importance prééminentel C'est encore le besoin de beauté, si profond qu’à le mépriser de façon prolongée, on peut avilir une âmel C'est le besoin de justice, si aigu dans les contestations ouvrières, et dont l'étude doit nécessairement être tentée, sinon même vécue, et de la façon la plus concrètel

Réalité spirituelle, enfin! Et c'est évidemment ici que l'introduction de la personne humaine comme hypothèse d'interprétation des phénomènes sociaux doit comporter le plus de fécondité. Nul ne conteste en effet, encore que sous des vocables différents, l'existence de la réalité biologique et de la réalité émotionnelle. Mais affirmer la réalité spirituelle de la personne humaine, voilà qui va engager, du même coup, dans une direction donnée, toutes les sciences sociales, embauchant la sociologie, l'économique et la politique au service de l'esprit, de même que les sciences biologiques sont édifiées pour être au service de la vie. Affirmer la réalité spirituelle de la personne humaine, c'est affirmer l'autonomie d'une conscience et d'une volonté, c'est affirmer une aptitude originale à la connaissance et à l'action. C'est donc affirmer la liberté métaphysique du choix, le libre arbitre humain.

\section{*** $*$}

D'aucuns penseront peut-être qu'il est bien audacieux de mettre ainsi la personne humaine au coeur de l'objet des sciences sociales, alors que tant de sociologues ont dit et répété que les faits sociaux étaient spécifiquement différents des faits individuels. C'est cependant en pleine connaissance de cause qu'après d'autres nous revenons sur ce point, car nous croyons précisément que la perspective proposée par un Emile Durkheim d'étudier les faits sociaux «comme des choses 》 n'a eu d'autre résultat que d'éliminer la liberté comme facteur d'explication des faits sociaux, et de rendre par là la science théoriquement séduisante et pratiquement stérile. Au contraire, à partir de la personne humaine, il semble que le sociologue soit à même de découvrir et d'interpréter, de façon ordonnée et satisfaisante, la totalité de la réalité sociale.

Et d'abord, la réalité familiale dont il faut reconnâ̂tre que certains sociologues ont gravement obscurci la notion. Ne voit-on pas, par exemple, Durkheim aller jusqu'à écrire que \&le clan est la famille par excellencel » De tels abus de langage ne sont pas à craindre si l'on élabore la sociologie de la famille sur une prise de conscience claire des personnes humaines qui contractent des liens conjugaux. Et il appartient au sociologue de déterminer ce qui relâche de tels liens et ce qui les resserre. Comment le ferait-il, si sa science accorde la même valeur au clan, à l'union libre et au mariage indissoluble?

De même, la réalité professionnelle présentera un tout autre visage selon qu'on l'étudiera comme un phénomène brut, ou que l'on évoquera la dignité et le destin de ceux qui la composent. Comme il est significatif, à ce propos, de voir, au même moment, l'industrie la plus moderne se heurter, sans en avoir encore clairement conscience, à ce problème de la personne humainel C'est chaque jour que depuis dix ans, aux Etats-Unis, les spécialistes des « human relations 》 voient leur importance s'accroître dans des proportions extraordinaires. C'est depuis presqu'aussi longtemps que l'on voit se développer en France de nombreuses initiatives privées et divers mouvements poursuivant un but similaire, et dont l'un d'cux porte le nom significatif d'<Economie et Humanisme». Et l'on pourrait multiplier les exemples.

De même encore, la réalité nationale se présentera différemment selon les catégories dans lesquelles on l'interprétera. Ce problème-là aussi est brûlant. Qu'il ait tendance à concevoir la liberté politique de façon un peu restrictive, ou qu'au contraire il interprète cette liberté de façon un peu lâche, et voilà que le sociologue, décrivant les mêmes faits, avec une méthode également rigoureuse, les interprètera différemment, et invitera donc à des interventions différentesl De même un marxiste et un libéral expliquent différemment le déterminisme de phénomènes sur le contenu desquels ils sont pourtant d'accord! Une exacte appréciation de la personne humaine sera donc, là encore, une règle très sûre pour prendre les mesures de la réalité politique.

L'explication sociologique de la communauté humaine prise comme un tout - cette famille des nations - ne peut, enfin, être féconde que dans la mesure où elle admet que tous les hommes du monde sont des personnes humaines. C'est évidemment faute de l'accepter, que les sociologues hitlériens ont pu décrire une réalité qui correspondait si parfaitement aux appétits de domination du régime. Et combien d'autres douloureux problèmes à l'ordre du jour seraient en voie de solution si, dans tous les pays du monde, chrétiens ou non, les observateurs sociaux s'entendaient simplement sur ce magnifique postulat, où nous chrétiens reconnaissons notre nature même, telle que Dieu nous l'a révélée: la personne humaine.

Au terme de ces quelques idées, qui n'ont d'autre portée que de condenser les réflexions d'un homme, qui, sil ne peut suivre de près l'activité des disciplines qu'il a évoquées, connaît un peu par expérience ce que des gouvernants conscients de leurs responsabilités attendent d'elles, comment ne pas se réjouir, en songeant que c'est cette science-là, dont l'acquisition est si importante dans notre monde troublé, que nos universités canadiennesfrançaises ont commencé à édifier. C'est notre vocation, à nous autres Canadiens, de définir, pour porter notre témoignage sur cette terre d'Amérique, ce que nous entendons être, afin de le vivre chaque jour plus profondément, et pour révéler aux autres hommes, nos frères, ce qu'ils sont - et qu'ils ignorent - . Pour cette tâche, nous disposons d'un pays riche, d'un peuple généreux, d'une situation économique enviable. Il nous reste à prouver que l'on peut bâtir un monde où la machine sera pour l'homme, et non l'homme pour la machine, où la production sera pour l'homme et non pas l'homme pour la production, où l'économie sera pour l'homme, et non pas l'homme pour l'économiel Quelles chances avonsnous donc de voir chez nous, puis autour de nous, grandir une civilisation qui protège et garantisse la personne? Parce que l'entreprise est osée, la dirons-nous téméraire? De toutes façons, c'est à nous qu'elle échoit. Et le monde qu'il faut construire ne peut plus être seulement à la taille de l'homme, car l'homme lui-même, aujourd'hui, est aux dimensions de l'univers. Le monde qu'il faut construire doit être à la taille du Prince de la Paix, de Cslui qui a dit: «Sans moi, vous ne pouvez rien fairel ? Mais avec lui, nous le tenteronsl 